\title{
Dark Radiative Inverse Seesaw Mechanism
}

\author{
Amine Ahriche* \\ The Abdus Salam International Centre for Theoretical Physics, Strada Costiera 11, I-34014, Trieste, Italy; \\ Laboratory of Mathematical and Sub-Atomic Physics (LPMPS), \\ University of Constantine I, DZ-25000 Constantine, Algeria; and \\ Department of Physics and Center for Theoretical Sciences, \\ National Taiwan University, Taipei 106, Taiwan \\ Sofiane M. Boucenna ${ }^{\dagger}$ \\ INFN, Laboratori Nazionali di Frascati, C.P. 13, 100044 Frascati, Italy. \\ Salah Nasri ${ }^{\ddagger}$ \\ Physics Department, UAE University, POB 17551, Al Ain, United Arab Emirates
}

\begin{abstract}
We present a minimal model that simultaneously accounts for neutrino masses and the origin of dark matter (DM) and where the electroweak phase transition is strong enough to allow for electroweak baryogenesis. The Standard Model is enlarged with a Majorana fermion, three generations of chiral fermion pairs, and a single complex scalar that plays a central role in DM production and phenomenology, neutrino masses, and the strength of the phase transition. All the new fields are singlets under the SM gauge group. Neutrino masses are generated via a new variant of radiative inverse seesaw where the required small mass term is generated via loops involving DM and no large hierarchy is assumed among the mass scales. The model offers all the advantage of low-scale neutrino mass models as well as a viable dark matter candidate that is testable with direct detection experiments.
\end{abstract}

\section{INTRODUCTION}

There are at least three concrete evidences which suggest that the Standard Model (SM) is incomplete. These are i) non-zero neutrino masses, $i i)$ the existence of dark matter (DM), and iii) the observation of the matterantimatter asymmetry of the universe. Neutrinos are usually assumed to be Majorana particles, in which case an understanding of the origin of their mass necessarily requires new degrees of freedom above the electroweak scale. Similarly, the explanation for the $27 \%$ [1] of the total energy density of the universe in the form of DM implies the need to extend the SM with at least one additional neutral particle that is stable on cosmological time scales. The reason for the fact that the observable universe is made of matter and not antimatter and that the value of the cosmic baryon-to-photon ratio (i.e, the baryon asymmetry of the Universe (BAU)) is about $6 \times 10^{-10}$ have no explanation within the SM either and require new physics. While many extensions of the SM exist to solve these problems individually, minimality as dictated by Occam's razor would suggest that models offering simultaneous explanations to neutrinos and dark matter [2-4], or dark matter and BAU [5-8], or the three at once are favored. It is our goal here to address simultaneously all these shortcomings of the SM in a unified framework at the $\mathrm{TeV}$ scale.

\footnotetext{
*Electronic address: aahriche@ictp.it

${ }^{\dagger}$ Electronic address: boucenna@lnf.infn.it

$\ddagger$ Electronic address: snasri@uaeu.ac.ae
}

The simplest mechanism for generating small neutrino masses is the seesaw (type-I) mechanism [9-13] where three massive right-handed neutrinos are coupled to the left handed neutrinos. However on the basis of naturalness, it invokes a right handed neutrino with mass of order of Grand Unified Theories (GUT) scale, making it hopeless to probe it in high energy physics experiments. One way to lower the scale of the new physics is by invoking 'low-scale mechanisms' [2], in particular the inverse seesaw where one extends the seesaw mechanism with additional singlet fermions, $N_{L}$, and arrange for the lepton charges such that the 2-units violation of lepton number resides in the singlet mass term $\mu N_{L} N_{L}$ [14]. The resulting light neutrino masses are linearly proportional to $\mu, m_{\nu} \sim\left(m_{D} / M\right)^{2} \mu$, with $m_{D}$ and $M$ the usual Dirac mass and New Physics (NP) scale respectively. It is clear then that if one chooses $\mu \sim \mathrm{keV}$, the scale of NP can be of order TeV. However, the smallness of $\mu$ remains unexplained although usually justified in terms of 't Hooft naturalness.

On the other hand, neutrino masses could be generated radiatively at a certain $n$-loop level. The idea is that their mass can be naturally small due to the loop suppression factor, $1 /\left(16 \pi^{2}\right)^{n}$, and the product of Yukawa couplings instead of a suppression by the NP scale [15-22] (for a review see [2]). This suppression allows the mass of the new particles involved in the generation of neutrino masses to be much smaller than the canonical seesaw mass scale. For instance, in the three-loop neutrino mass generation models, the scale of the new particles can be in the hundreds $\mathrm{GeV}$ scale, 


\begin{tabular}{c|c|c|cc}
\hline & $L, \ell_{R}, N_{R}, N_{L}$ & $\chi$ & $H$ & $S$ \\
\hline $\mathbb{Z}_{4}$ & $i$ & -1 & +1 & $i$ \\
\hline
\end{tabular}

TABLE I: Summary of the relevant fields of the model and their quantum numbers.

which makes them testable at collider experiments [23-26]. Furthermore, the use of discrete symmetry that precludes the tree-level mass term for neutrinos, allows the existence of DM candidate ${ }^{1}$ which plays a role in the radiative neutrino mass generation [28, 29], and could also trigger the electroweak symmetry breaking [30, 31].

In this work we propose a simple radiative inverse seesaw model where we extend the SM with three chiral fermions and one complex scalar field that are all singlet under the SM gauge group. A $\mathbb{Z}_{4}$ symmetry is invoked to simultaneously forbid the tree level inverse seesaw contribution and provide a stable DM candidate. The $\mu$ term is induced radiatively via DM particles circulating in a loop. In this model all the exotic particles have masses of order $\mathrm{TeV}$ scale or less, which makes them accessible for collider experiments. The observed DM relic density can be naturally obtained and the spin-independent scattering cross section of the DM off nucleus is consistent with the experimental limit reported by LUX [32], and yet within the reach of future DM direct detection searches. In addition, a strongly first order electroweak phase transition can be achieved, which is required for a successful implementation of electroweak baryogenesis [33]. We refer to Refs. [34-40] for other radiative or linear inverse seesaw constructions.

This paper is organized as follows. In section (II) we present the model. The generation of neutrino mass is presented in section (III). In section (IV) we study the phenomenology of the scalar sector and the strength of the electroweak phase transition. The calculation of the DM relic abundance and direct detection is discussed in section (V). Finally, we give our conclusion in section (VI).

\section{THE MODEL}

We consider a simple extension of the SM by adding three generations ${ }^{2}$ of chiral fermion pairs $N_{R}$ and $N_{L}$, one other chiral fermion $\chi_{R} \equiv \chi$ and a complex scalar $S$. All the new fields transform trivially under the SM

\footnotetext{
${ }^{1}$ A generalization of [20] with septuplet representations [27] has the interesting feature of automatically containing stable DM candidate, without requiring a new discrete symmetry.

${ }^{2}$ For simplicity we add the iso-singlet pairs sequentially, though two pairs would suffice to account for the neutrino oscillations data.
}

gauge group, however we assign different charges to the fields of the model under an imposed $\mathbb{Z}_{4}$ symmetry (or similarly, different $B-L$ charges), c.f. table (I). The SM quark sector is left unchanged.

The relevant terms in the Yukawa Lagrangian are the following (flavor indices are omitted):

$$
\begin{aligned}
-\mathcal{L} \supset & y_{\nu} \bar{L} \tilde{H} N_{R}+M \overline{N_{L}} N_{R} \\
& +y_{N} S \bar{\chi} N_{L}+\frac{m_{\chi}}{2} \chi^{T} C^{-1} \chi+\text { h.c. }
\end{aligned}
$$

where $\tilde{H} \equiv i \sigma_{2} H^{\star}$ and $C$ is the charge conjugation operator. The scalar potential is given by:

$$
\begin{aligned}
V= & -\mu_{H}^{2} H^{\dagger} H+\frac{1}{2} \lambda_{H}\left(H^{\dagger} H\right)^{2} \\
& +\mu_{S}^{2} S^{\star} S+\frac{\mu_{\nu}^{2}}{2}\left(S^{2}+\text { h.c. }\right)+\frac{\lambda_{S}}{2}\left(S^{\star} S\right)^{2} \\
& +\lambda_{H S} H^{\dagger} H S^{\star} S .
\end{aligned}
$$

The term $\mu_{\nu}^{2}$ which breaks the $\mathbb{Z}_{4}$ symmetry softly is required by neutrino masses, as it is the origin of lepton number violation (by two units). This will become clear in section (III). We see this term as a low energy manifestation of an ultra-violet completion of the model and we remain agnostic as to its specific origin. This could be for instance, a result of a hidden sector that couples to the visible sector via the 'super-renormalizable' terms of the singlet $S$ [41]. The mixed quartic coupling $\lambda_{H S}$ has to be positive because we found that negative values destabilize the potential not far from the EW scale. With positive $\lambda_{H S}$ we always found stability up to at least $10^{6}$ $\mathrm{GeV}$, where the model is completed by a more complete theory. In the next section we address the neutrino phenomenology of the model.

\section{NEUTRINO MASSES}

The neutrino mixing matrix in the basis $N^{T}=$ $\left(\nu_{L}, N_{R}^{c}, N_{L}, \chi^{c}\right)$, the neutral fermions mass term of the form $\frac{1}{2} \overline{N^{c}} \mathcal{M} N+$ H.c. is:

$$
\mathcal{M}=\left(\begin{array}{cccc}
0 & m_{D}^{\top} & 0 & 0 \\
m_{D} & \epsilon_{R} & M & 0 \\
0 & M^{\top} & \epsilon_{L} & 0 \\
0 & 0 & 0 & m_{\chi}
\end{array}\right)
$$

where $m_{D}=y_{\nu}\langle H\rangle$ is the usual Dirac neutrino mass.

The terms $\epsilon_{L, R}$ are generated radiatively in this model; the loop contribution is made possible thanks to the presence of the coupling $y_{N} N_{L} \bar{\chi} S$ in eq. (1), the soft breaking term in eq. (2) which allows for the chirality flip, and the condition $\langle S\rangle=0$ which forbids the tree-level contribution. In the tree-level limit, i.e., $\epsilon_{R, L}=0$, we have three strictly massless neutrinos and three heavy Dirac pairs of neutrinos - lepton number is a good symmetry of the Lagrangian. However, by turning on the terms $\epsilon_{R, L}$, the 

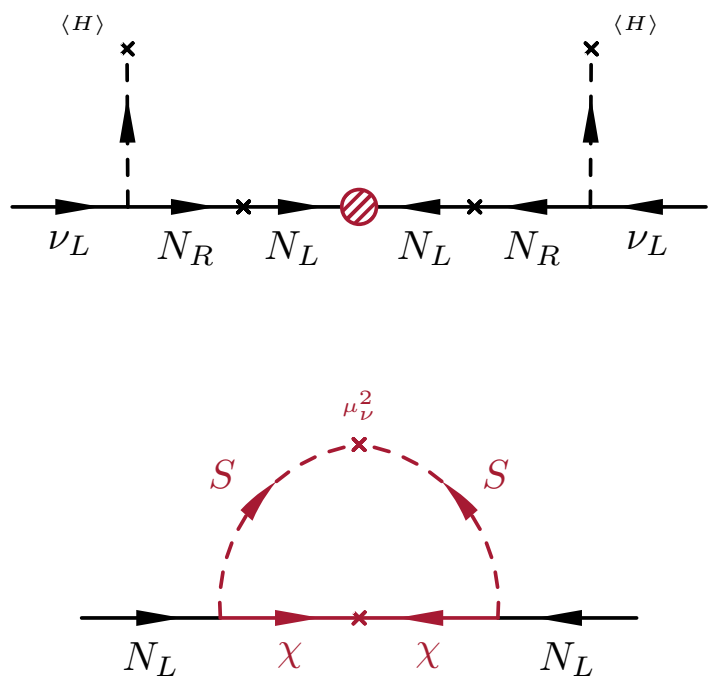

FIG. 1: Radiative inverse seesaw with DM.

additive conservation of lepton number gets violated and induces small neutrino masses:

$$
m_{\nu} \simeq m_{D}^{\top} \frac{1}{M^{\top}} \epsilon_{L} \frac{1}{M} m_{D} \equiv m_{D}^{\top} \mathcal{M}_{R}^{-1} m_{D},
$$

at lowest order in $\epsilon_{R, L}$. The neutrino mass contribution from $\epsilon_{R}$ gets an additional loop suppression with respect to that of $\epsilon_{L}$ [42] and so we will ignore it here. The light neutrino masses are linearly proportional to $\epsilon_{L}$, which is the term responsible of lepton number violation. Based on this, one can argue that $\epsilon_{L}$ should be small because in its absence the symmetry of the theory is enhanced; this is 'technical naturalness' in the 't Hooft sense. Whereas in most models invoking the inverse seesaw mechanism to generate neutrino masses $\epsilon_{L}$ is assumed to be tiny, here this is justified by the fact that it is generated radiatively via a loop which involves our DM candidate.

The matrix $\epsilon_{L}$ is induced by the diagrams in fig. (1), and is found to be:

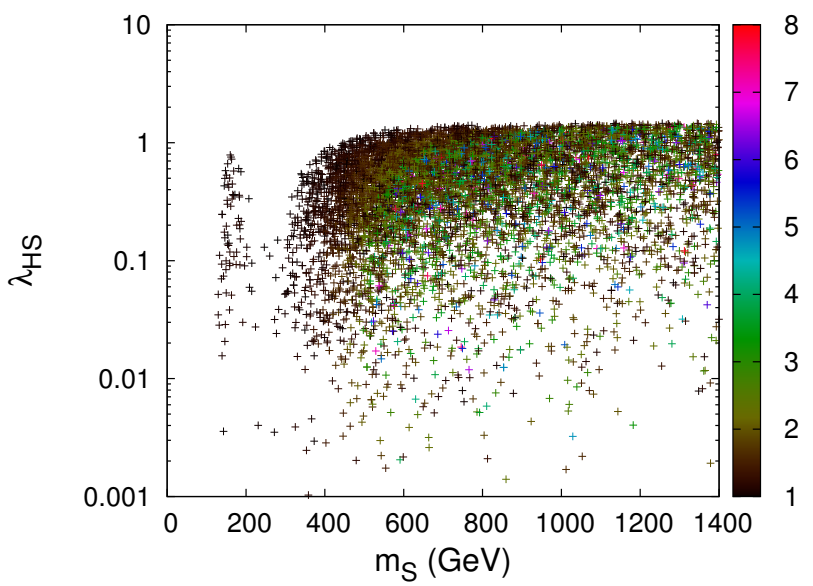

FIG. 2: The coupling $\lambda_{H S}$ in absolute value versus the scalar mass, and the palette reads the phase transition strength, eq. (19).

Neutrino masses and mixing angles can be accommodated for a given $\epsilon_{L}$, that is for a set of parameters $\left\{m_{S}, m_{\chi}, y_{N}, \mu_{\nu}\right\}$, by using the freedom we have on the neutrino Yukawa coupling. Assuming $\mathcal{M}_{R}$ to be diagonal, the Yukawa couplings appearing in $m_{D}$ can be parameterized as [55]:

$$
y_{\nu}=\sqrt{\mathcal{M}_{R}} \mathcal{R} \sqrt{\hat{m}_{\nu}} U_{l e p}^{\dagger},
$$

where $U_{l e p}$ is the lepton mixing matrix, $U_{l e p}^{\top} m_{\nu} U_{l e p}=$ $\operatorname{diag}\left(m_{1}, m_{2}, m_{3}\right) \equiv \hat{m}_{\nu}\langle H\rangle^{2}$, and $\mathcal{R}$ is an orthogonal matrix. Because of this freedom, we find that the neutrino parameters as well as limits on unitarity deviations and flavor changing currents can easily be accommodated.

\section{HIGGS PHYSICS AND ELECTROWEAK PHASE TRANSITION}

We can get naturally small values for $\epsilon_{L}$ without having to put by hand a number which is far from the weak scale in the Lagrangian. For instance, for $\mu_{\nu}=10 \mathrm{GeV}$, $m_{\chi}=100 \mathrm{GeV}$, and $m_{S}=1 \mathrm{TeV}$, we get $\epsilon_{L} \approx 3 \mathrm{keV}$. With the additional freedom introduced by $\epsilon_{L}$, neutrino masses become decoupled from the mixing between light and heavy neutrinos and therefore from the strength of lepton flavor violation [43]. This makes such a framework particularly rich phenomenologically, as it leads to many signals at low energy physics experiments (see, e.g., [4447]) as well as high energy colliders (e.g., [48-54]).

\section{Higgs Mass}

The Higgs mass at one-loop can be estimated as the second derivative of the zero-temperature effective potential that is given in the $\overline{D R}^{\prime}$ scheme by [56]:

$$
\begin{aligned}
V_{1-l}^{T=0}(h)= & -\frac{\mu_{H}^{2}}{2} h^{2}+\frac{\lambda_{H}}{8} h^{4} \\
& +\sum_{i} n_{i} \frac{m_{i}^{4}(h)}{64 \pi^{2}}\left(\log \frac{m_{i}^{2}(h)}{\Lambda^{2}}-\frac{3}{2}\right),
\end{aligned}
$$



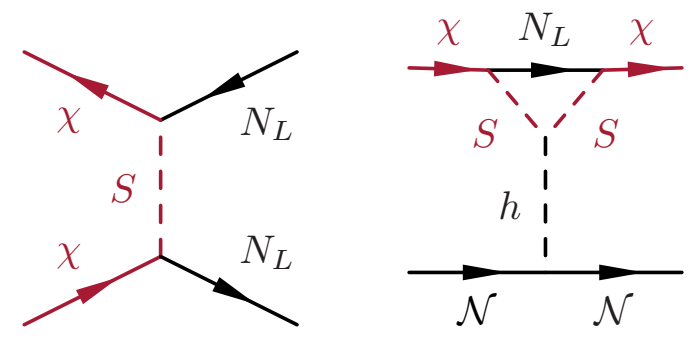

FIG. 3: DM production (left) and direct detection (right) diagrams.

where $h=\left(\sqrt{2} \Re\left(H^{0}\right)-v\right)$ is the real part of the neutral component in the doublet, $n_{i}$ are the field multiplicity, $\Lambda$ is the renormalization scale which we choose to be the Higgs mass, $125 \mathrm{GeV}$, and $m_{i}^{2}(h)$ are the field-dependent mass squared that can be written as $m_{i}^{2}(h)=\alpha_{i}+\frac{1}{2} \beta_{i} h^{2}$, i.e.,

$$
\begin{aligned}
m_{W}^{2}(h) & =\frac{1}{4} g^{2} h^{2} \\
m_{Z}^{2}(h) & =\frac{1}{4}\left(g^{2}+g^{2}\right) h^{2} \\
m_{t}^{2}(h) & =\frac{1}{2} y_{t}^{2} h^{2} \\
m_{h}^{2}(h) & =-\mu_{H}^{2}+\frac{3}{2} \lambda_{H} h^{2} \\
m_{G}^{2}(h) & =-\mu_{H}^{2}+\frac{1}{2} \lambda_{H} h^{2} \\
m_{S_{r, i}}^{2}(h) & =\mu_{S}^{2}+\frac{1}{2} \lambda_{H S} h^{2} \pm \frac{1}{2} \mu_{\nu}^{2},
\end{aligned}
$$

where $G$ and $S_{r} \equiv \Re(S) \quad\left(S_{i} \equiv \Im(S)\right)$ denote the Goldstone bosons and the real (imaginary) part of the complex scalar $S$, respectively. In all what follows we will consider the case $\mu_{S}^{2}+\frac{1}{2} \lambda_{H S} v^{2} \gg \frac{1}{2} \mu_{\nu}^{2}$, such that $m_{S} \equiv m_{S_{r}} \sim m_{S_{i}}$.

The term $\mu_{H}^{2}$ can be eliminated in favor of the doublet vacuum expectation value (vev) via the tadpole condition at one-loop:

$$
\mu_{H}^{2}=\frac{1}{2} \lambda_{H} v^{2}+\frac{1}{32 \pi^{2}} \sum_{i} n_{i} \beta_{i} m_{i}^{2}\left(\log \frac{m_{i}^{2}}{\Lambda^{2}}-1\right),
$$

with $\dot{m}_{i}^{2}(h)=\partial m_{i}^{2}(h) / \partial h$ and by using eq. (9), the Higgs mass squared can be written as:

$$
m_{h}^{2}=\lambda_{H} v^{2}+\frac{v^{2}}{32 \pi^{2}} \sum_{i} n_{i} \beta_{i}^{2} \log \frac{m_{i}^{2}}{\Lambda^{2}} .
$$

In order to explain the discovered scalar resonance at $m_{h}=125.09 \mp 0.21 \mathrm{GeV}[57,58]$, the Higgs quartic coupling $\lambda_{H}$ has to be adjusted according to the radiative corrections.

\section{Higgs Invisible Decay}

If either of $m_{S}$ or $m_{\chi}$ is smaller than half of the Higgs mass then the bounds on Higgs invisible decay should be fulfilled, i.e., $\mathcal{B}(h \rightarrow$ inv $)<17 \%$ [59]. Here, the Higgs invisible decay branching ratio is given by:

$$
\mathcal{B}(h \rightarrow i n v)=\frac{\sum_{X} \Gamma\left(h \rightarrow X X^{c}\right)}{\sum_{X} \Gamma\left(h \rightarrow X X^{c}\right)+\Gamma_{\mathrm{SM}}},
$$

where $X \equiv \chi, S_{r}, S_{i}$ and $\Gamma_{\mathrm{SM}}=4.434 \mathrm{MeV}$ is the $\mathrm{SM}$ Higgs decay width. The Higgs decay widths to $S$ and $\chi$ are given by:

$$
\begin{aligned}
& \Gamma\left(h \rightarrow S S^{\star}\right)=\frac{\lambda_{H S}^{2} v^{2}}{16 \pi m_{h}}\left(1-\frac{4 m_{S}^{2}}{m_{h}^{2}}\right)^{\frac{1}{2}} \Theta\left(m_{h}-2 m_{S}\right), \\
& \Gamma\left(h \rightarrow \chi \chi^{c}\right)=\frac{y_{\chi}^{2}}{16 \pi} m_{h}\left(1-\frac{4 m_{\chi}^{2}}{m_{h}^{2}}\right)^{\frac{3}{2}} \Theta\left(m_{h}-2 m_{\chi}\right) .
\end{aligned}
$$

The decay to $\chi$ occurs through the triangle one-loop vertex $y_{\chi} h \bar{\chi} \chi$, that is shown in fig. (3). This effective vertex is given in terms of the parameters of the model by:

$$
y_{\chi}=\frac{\lambda_{H S} v}{16 \pi^{2} m_{S}^{2}} \sum_{i} y_{N_{i}}^{2} M_{i} Q\left(\frac{M_{i}^{2}}{m_{S}^{2}}\right)
$$

where the function $Q$ is defined as:

$$
Q(x)= \begin{cases}Q^{+}(x) & x>\frac{1}{4} \\ Q^{-}(x) & x<\frac{1}{4}\end{cases}
$$

with

$$
\begin{aligned}
& Q^{+}(x)=\frac{2}{\sqrt{y}}\left[\arctan \left(\frac{2 x-1}{\sqrt{y}}\right)+\arctan \left(\frac{1}{\sqrt{y}}\right)\right] \\
& Q^{-}(x)=\frac{1}{\sqrt{-y}}\left(\log \left(\frac{2 x-1-\sqrt{-y}}{2 x-1+\sqrt{-y}}\right)-\log \left(\frac{1+\sqrt{-y}}{1-\sqrt{-y}}\right)\right)
\end{aligned}
$$

where $y=4 x-1$.

When only the $S$ channel is open, this constraint can be translated as an upper bound on the quartic coupling:

$$
\lambda_{H S}\left(1-\frac{4 m_{S}^{2}}{m_{h}^{2}}\right)^{1 / 4} \lesssim 9.7 \times 10^{-3} .
$$

\section{Electroweak Phase Transition}

The SM has all the qualitative ingredients for electroweak baryogenesis, however the generated matter-antimatter asymmetry cannot account for observations due the smallness of $C P$ violation and the fact that electroweak phase transition (EWPT) is not strongly first order [33]. However, it is well known that the EWPT can be strengthened if new scalar degrees of freedom coupled to the Higgs are added [60-67]. In this model, we have two extra scalar degrees of freedom around the weak scale, 


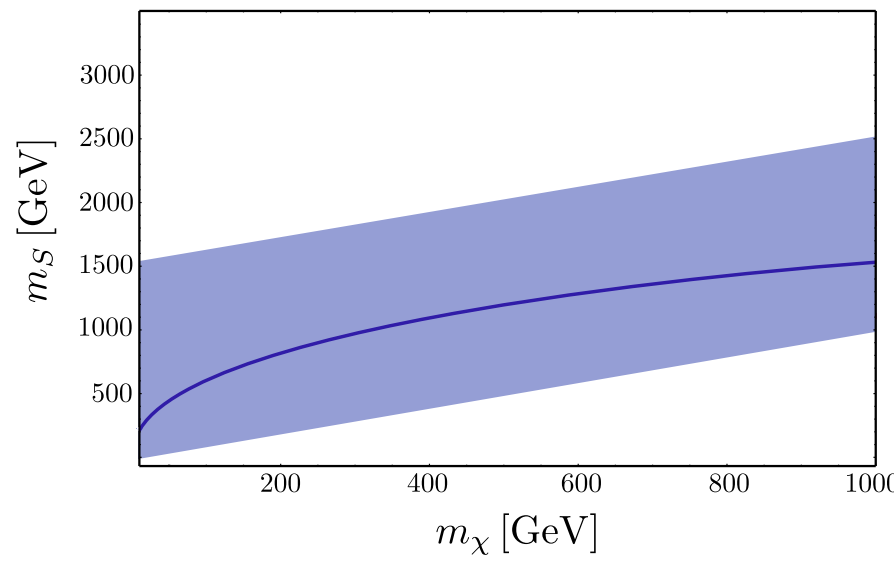

FIG. 4: The mediator mass as a function of the DM mass. All the benchmark points satisfy the relic density abundance and the detection limits. The solid line is the particular benchmark defined by $y_{N}=1$ and $M_{1}=10 \mathrm{GeV}$.

so we expect an enhancement of the EWPT strength.

In order to investigate the nature of the EWPT, the effective potential should be properly defined at finite temperature. The full effective potential can be written as $[68,69]$ :

$$
\begin{aligned}
V_{e f f}(h, T) & =V_{1-l}^{T=0}(h)+\frac{T^{4}}{2 \pi^{2}} \sum_{i} n_{i} J\left(\frac{m_{i}(h)^{2}}{T^{2}}\right), \\
J(\beta) & =\int_{0}^{\infty} x \log \left(1+\eta \exp ^{-\sqrt{x^{2}+\beta}}\right) d x,
\end{aligned}
$$

with $\eta=-1(+1)$ for bosons (fermions). Another bosonic thermal contribution should be included in eq. (18). This contribution represents a leading part of higher order corrections that is estimated by performing the resummation of an infinite class of infrared-divergent multi-loops, known as the ring (or daisy) diagrams [70]. We will include this effect by replacing the field-dependent masses of the scalar and longitudinal degrees of freedom by their thermal corrections $\tilde{m}_{i}^{2}(h, T)=m_{i}^{2}(h)+\Pi_{i}(T)$, where the thermal parts are given by:

$$
\begin{aligned}
\Pi_{W}^{L} & =\frac{11}{6} g^{2} T^{2}, \quad \Pi_{W}^{\top}=\Pi_{Z}^{\top}=0, \quad \Pi_{B}=\frac{11}{16} g^{\prime 2} T^{2}, \\
\Pi_{h} & =\Pi_{\chi}=\left(\frac{1}{2} \lambda_{H}+\frac{1}{6} \lambda_{H S}+\frac{1}{4} g^{2}+\frac{1}{12} g^{2}+\frac{1}{4} y_{t}^{2}\right) T^{2}, \\
\Pi_{S} & =\left(\frac{1}{3} \lambda_{S}+\frac{1}{3} \lambda_{H S}\right) T^{2},
\end{aligned}
$$

with $g, g^{\prime}$ and $y_{t}$ are the gauge and Yukawa couplings.

To generate a net baryon asymmetry at the electroweak scale [71], the anomalous interactions that violate $B+$ $L$ should be switched-off inside the nucleated bubbles, which leads to the strong first order phase transition criterion [72]

$$
v_{c} / T_{c}>1
$$

where $T_{c}$ is the critical temperature at which the effective potential exhibits two degenerate minima, and $v_{c}$ is the Higgs doublet vev at this temperature. The transition can be defined by two conditions:

$$
\left.\frac{\partial}{\partial h} V_{e f f}\left(h, T_{c}\right)\right|_{h=v_{c}}=0, \quad V_{e f f}\left(v_{c}, T_{c}\right)=V_{e f f}\left(0, T_{c}\right)
$$

In the $\mathrm{SM}$ the criterion $v_{c} / T_{c} \sim$ $\left(2 m_{W}^{3}+m_{Z}^{3}\right) /\left(\lambda_{H} v^{3}\right)>1$ implies that the Higgs mass has to be $m_{h}<42 \mathrm{GeV}$ [73] in contradiction with measurements. Therefore in the SM, the electroweak phase transition is a smooth crossover. However, if the radiative contributions in the Higgs mass (second term in LHS of eq. (10)) are significant, the doublet quartic coupling $\lambda_{H}$ gets smaller and the phase transition gets stronger. We perform a random scan in the parameter space with 6000 benchmark points taking into account the Higgs mass and its branching ratio to invisibles. Our results are shown in fig. (2).

It is clear from fig. (2) that for the phase transition to be naturally strong, the mixed quartic coupling must satisfy $\lambda_{H S} \gtrsim 10^{-2}$, which means, after using eq. (16), that the invisible Higgs decay channel-barring tuning of parameters - must be closed, i.e., the scalar $S$ mass should be larger than $m_{h} / 2$. One also remarks that, in any case, a strong phase transition favors heavy scalars. Similar behavior had been seen in $[65,74-78]$, where extra scalars can help bring about a strongly first-order EWPT by: (a) reducing the Higgs quartic coupling $\lambda_{H}$ to small values and having significant radiative correction to get the correct Higgs mass, eq. (10); and (b) enhancing the value of the effective potential at the wrong vacuum at the critical temperature without suppressing the ratio $v_{c} / T_{c}$, which relaxes the severe bound on the mass of the SM Higgs.

At the International Linear Collider (ILC), the triple Higgs coupling $\lambda_{h h h}$ can be measured with about $20 \%$ accuracy or better at $\sqrt{s}=500 \mathrm{GeV}$ with integrated luminosity $\mathcal{L}=500 \mathrm{fb}^{-1}$ [79]. Unfortunately, within our numerical scan, one remarks that the relative enhancement in the triple Higgs model with respect to the SM,

$$
\Delta=\frac{\lambda_{h h h}-\lambda_{h h h}^{S M}}{\lambda_{h h h}^{S M}},
$$

lies between $-2.3 \%$ and $10 \%$.

One has to notice that an extra $C P$-violating source is required to have a realistic electroweak baryogenesis scenario. Therefore a $C P$-violating phase should be added in the Lagrangian of the complete theory. In analogy to a scenario of electroweak baryogenesis from a singlet scalar, one can modify the top quark Lagrangian mass term by adding a non-renormalizable dimension 6 operator where the complex scalar couples to the top-quark, 
for instance [80]

$$
\frac{e^{i \alpha}}{\Lambda^{2}} Q_{L} H t_{R} S^{2}
$$

This new interaction is suppressed by a new-physics scale that can be well above one $\mathrm{TeV}$.

\section{RELIC DENSITY ABUNDANCE AND DIRECT DETECTION}

The DM candidate can be either the fermion, $\chi$ or the lightest of the scalars $\Re(S)$ and $\Im(S)$ depending on the sign of $\mu_{\nu}$. The fermionic case has more predictive power because there are fewer production channels and the parameters entering the evaluation of the relic density are directly related to those entering in the neutrino masses. It is conceptually more elegant than the scalar cases whose phenomenology would be dependent upon the Higgs portal with no direct relation with neutrino masses. For these reasons we consider our dark matter candidate to be $\chi$.

In the non-relativistic limit, the thermally averaged annihilation cross-section can be written as $\left\langle\sigma v_{r}\right\rangle=a+b v_{r}^{2}$, where $v_{r} \simeq \sqrt{6 / x_{f}}$ is the relative DM velocity and $a$ and $b$ are respectively the $s$-wave and $p$-wave factors which receive contributions from different annihilation channels. The relic density is then given by

$$
\Omega h^{2} \simeq \frac{1.04 \times 10^{9} \mathrm{GeV}^{-1}}{M_{P l}} \frac{x_{f}}{\sqrt{g_{*}\left(T_{f}\right)}\left(a+3 b / x_{f}\right)},
$$

where $T_{f}=m_{\chi} / x_{f}$ is the freeze-out temperature, $g_{*}(T)$ is the number of relativistic species at temperature $T$, and $M_{P l}$ is the Planck mass.

The dark matter production is thermal via the freeze-out mechanism, and proceeds via the annihilation diagrams depicted in fig. (3). This implies that at least one of the heavy neutrinos is lighter than the DM. We will consider the hierarchies $M_{1}=M_{2} \equiv M_{N} \leq m_{\chi}$ and $M_{3}>1 \mathrm{TeV}$. $M_{i}$ are the diagonal entries of the matrix $M$ appearing in eq. (1). This hierarchy is found to satisfy the neutrino oscillation data and flavor-changing limits for any mass $m_{\chi}$ in the range $\mathrm{GeV}-\mathrm{TeV}$.

In the limit $m_{S} \gg m_{\chi} \gg M_{N}$, the annihilation cross section $\chi \chi \rightarrow N_{L_{i}} N_{L_{j}}$ is given by

$$
\langle\sigma v\rangle \simeq \frac{y_{N_{i}}^{2} y_{N_{j}}^{2} m_{\chi}^{2}}{48 \pi m_{S}^{4}} v_{r}^{2}
$$

and using, eq. (24) and eq. (23), the good relic abundance is obtained for

$$
\frac{\Omega h^{2}}{0.12} \simeq\left(\frac{x_{f}}{20}\right)\left(\frac{1}{y_{N}}\right)^{4}\left(\frac{500 \mathrm{GeV}}{m_{\chi}}\right)^{2}\left(\frac{m_{S}}{\mathrm{TeV}}\right)^{4} .
$$

Therefore, we see that the model quite naturally reproduces the relic density constraint. In order to cover

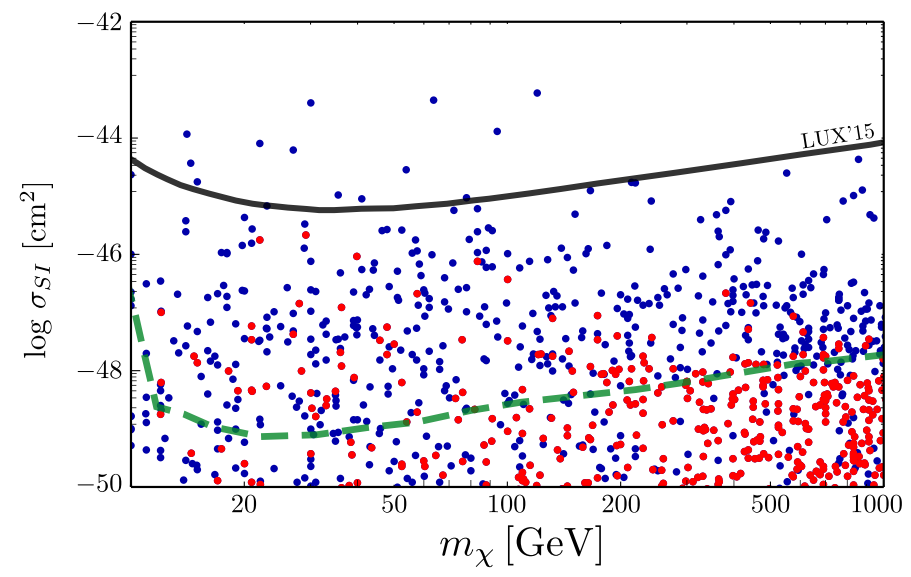

FIG. 5: Direct detection cross section versus the DM mass. Solid line is the limit from LUX experiment [32] and the dashed line is the neutrino floor. The points in red satisfy the conservative bound on the couplings $\left|y_{N_{i}}\right|, \lambda_{H S}<1$.

the full parameter space, we implemented the model in micrOmegas [81]. ${ }^{3}$ We perform a random scan on the relevant parameters of the model in the following ranges:

$$
\begin{aligned}
10 \mathrm{GeV} & \leq m_{\chi} \leq 1 \mathrm{TeV} \\
1 \mathrm{GeV} & \leq M_{N} \leq 1.05 m_{\chi} \\
1 \mathrm{TeV} & \leq M_{3} \leq 2.5 \mathrm{TeV} \\
m_{h} / 2 & <m_{S} \leq 1.5 \mathrm{TeV} \\
10^{-4} & \leq\left|y_{N_{i}}\right|, \lambda_{H S} \leq \sqrt{4 \pi}
\end{aligned}
$$

and we fix $\mu_{\nu}=1 \mathrm{GeV}$. For simplicity we take $y_{N_{1}}=y_{N_{2}} \equiv y_{N}$.

In fig. (4), we show the allowed parameter space in the plane $m_{S}$ versus DM mass, $m_{\chi}$. The solid line represents the evolution of the particular benchmark model defined by $y_{N}=1$ and $M_{1}=10 \mathrm{GeV}$, which confirms the estimate in eq. (25). All the points in fig. (4) and fig. (5) satisfy the relic density constraint [1]

$$
\Omega h^{2}=0.1198 \pm 0.0015
$$

as well as the requirement for a strong first order phase transition, eq. (19), and the bound on Higgs invisible decay.

The direct detection is obtained via the radiative diagram shown in fig. (3). We have implemented the effective interaction vertex, eq. (14), in our model. In fig. (5), we show the expected spin-independent cross section as a function of the DM mass for the range of parameters defined in eq. (26). We show also the points

\footnotetext{
${ }^{3}$ We used the FeynRules [82] package to generate the model file.
} 
which satisfy a more conservative bound on the couplings, $\left|y_{N_{i}}\right|, \lambda_{H S}<1$. The solid line is the current best limit on direct detection experiments, obtained from the latest results of the LUX experiments [32] which are the strongest to date. The dashed line illustrates the 'neutrino floor'. As apparent from the plot, the model is already probed by direct detection experiments even thought the scattering is loop-suppressed, and future runs of current experiments as well as planned experiments will probe a significant portion of the parameter space of the model.

\section{CONCLUSIONS}

In this paper, we have presented a model which provides simultaneous explanations for neutrino masses and dark matter. Neutrino oscillations are accounted for thanks to a DM-assisted radiative inverse seesaw mechanism, where the small lepton number violating parameter is generated at the one-loop level. There is no assumed hierarchy in the mass scales of the model and they can all be
$\mathcal{O}(\mathrm{GeV}-\mathrm{TeV})$. The symmetry which precludes the treelevel inverse seesaw contribution provides at the same time a fermionic dark matter candidate whose abundance is consistent with cosmological data and its scattering cross section off nuclei satisfies the latest LUX bound and can be probed by future DM direct detection experiments. The scalar responsible of the DM interactions with the visible sector as well as the generation of neutrino masses triggers a strong enough electroweak phase transition to make electroweak baryogenesis viable.

Acknowledgment AA is supported by the Algerian Ministry of Higher Education and Scientific Research under the CNEPRU Project No. D01720130042. SMB thanks F. Deppisch for discussions and acknowledges financial support from the research grant "Theoretical Astroparticle Physics" number 2012CPPYP7 under the program PRIN 2012 funded by the Italian MIUR and from the INFN "IS" Theoretical Astroparticle Physics (TAsP-LNF) and support of the spanish MICINN's Consolider-Ingenio 2010 Programme under grant MultiDark CSD2009-00064.
[1] P. A. R. Ade et al. (Planck) (2015), 1502.01589.

[2] S. M. Boucenna, S. Morisi, and J. W. F. Valle, Adv. High Energy Phys. 2014, 831598 (2014), 1404.3751.

[3] D. Restrepo, O. Zapata, and C. E. Yaguna, JHEP 1311, 011 (2013), 1308.3655.

[4] M. Lattanzi, R. A. Lineros, and M. Taoso, New J. Phys. 16, 125012 (2014), 1406.0004.

[5] S. M. Boucenna and S. Morisi, Front. Phys. 1, 33 (2014), 1310.1904.

[6] H. Davoudiasl and R. N. Mohapatra, New J. Phys. 14, 095011 (2012), 1203.1247.

[7] K. Petraki and R. R. Volkas, Int. J. Mod. Phys. A28, 1330028 (2013), 1305.4939.

[8] K. M. Zurek, Phys. Rept. 537, 91 (2014), 1308.0338.

[9] P. Minkowski, Phys. Lett. B67, 421 (1977).

[10] T. Yanagida, Conf.Proc. C7902131, 95 (1979).

[11] R. N. Mohapatra and G. Senjanovic, Phys. Rev. Lett. 44, 912 (1980).

[12] M. Gell-Mann, P. Ramond, and R. Slansky, Conf.Proc. C790927, 315 (1979), 1306.4669.

[13] J. Schechter and J. W. F. Valle, Phys. Rev. D22, 2227 (1980).

[14] R. N. Mohapatra and J. W. F. Valle, Phys. Rev. D34, 1642 (1986).

[15] T. P. Cheng and L.-F. Li, Phys. Rev. D22, 2860 (1980).

[16] A. Zee, Phys. Lett. B93, 389 (1980).

[17] E. Ma, Phys. Rev. Lett. 81, 1171 (1998), hep$\mathrm{ph} / 9805219$.

[18] A. Zee, Nucl. Phys. B264, 99 (1986).

[19] K. S. Babu, Phys. Lett. B203, 132 (1988).

[20] L. M. Krauss, S. Nasri, and M. Trodden, Phys.Rev. D67, 085002 (2003), hep-ph/0210389.

[21] M. Aoki, S. Kanemura, and O. Seto, Phys. Rev. Lett. 102, 051805 (2009), 0807.0361.

[22] M. Gustafsson, J. M. No, and M. A. Rivera,
Phys.Rev.Lett. 110, 211802 (2013), 1212.4806.

[23] A. Arhrib, C. Bhm, E. Ma, and T.-C. Yuan (2015), 1512.08796

[24] K. S. Babu and C. Macesanu, Phys. Rev. D67, 073010 (2003), hep-ph/0212058.

[25] A. Ahriche, S. Nasri, and R. Soualah, Phys. Rev. D89, 095010 (2014), 1403.5694.

[26] M. Aoki, S. Kanemura, and O. Seto, Phys.Rev. D80, 033007 (2009), 0904.3829.

[27] A. Ahriche, K. L. McDonald, S. Nasri, and T. Toma, Phys. Lett. B746, 430 (2015), 1504.05755.

[28] E. Ma, Phys.Rev. D73, 077301 (2006), hep-ph/0601225.

[29] A. Ahriche, C.-S. Chen, K. L. McDonald, and S. Nasri, Phys. Rev. D90, 015024 (2014), 1404.2696.

[30] A. Ahriche, K. L. McDonald, and S. Nasri, JHEP 10, 167 (2014), 1404.5917.

[31] A. Ahriche, K. L. McDonald, and S. Nasri (2015), 1508.02607.

[32] D. S. Akerib et al. (LUX) (2015), 1512.03506.

[33] M. Trodden, Rev. Mod. Phys. 71, 1463 (1999), hep$\mathrm{ph} / 9803479$.

[34] E. Ma, Phys. Rev. D80, 013013 (2009), 0904.4450.

[35] S. S. C. Law and K. L. McDonald, Phys. Lett. B713, 490 (2012), 1204.2529

[36] H. Okada and T. Toma, Phys. Rev. D86, 033011 (2012), 1207.0864

[37] G. Guo, X.-G. He, and G.-N. Li, JHEP 10, 044 (2012), 1207.6308 .

[38] I. Baldes, N. F. Bell, K. Petraki, and R. R. Volkas, JCAP 1307, 029 (2013), 1304.6162.

[39] S. Fraser, E. Ma, and O. Popov, Phys. Lett. B737, 280 (2014), 1408.4785.

[40] W.-C. Huang and F. F. Deppisch, Phys. Rev. D91, 093011 (2015), 1412.2027.

[41] B. Patt and F. Wilczek (2006), hep-ph/0605188. 
[42] P. S. B. Dev and A. Pilaftsis, Phys. Rev. D86, 113001 (2012), 1209.4051.

[43] J. Bernabeu, A. Santamaria, J. Vidal, A. Mendez, and J. W. F. Valle, Phys. Lett. B187, 303 (1987).

[44] M. Malinsky, T. Ohlsson, Z.-z. Xing, and H. Zhang, Phys. Lett. B679, 242 (2009), 0905.2889.

[45] D. V. Forero, S. Morisi, M. Tortola, and J. W. F. Valle, JHEP 09, 142 (2011), 1107.6009.

[46] R. Lal Awasthi and M. K. Parida, Phys. Rev. D86, 093004 (2012), 1112.1826.

[47] S. Antusch and O. Fischer, JHEP 10, 094 (2014), 1407.6607.

[48] F. Deppisch, T. S. Kosmas, and J. W. F. Valle, Nucl. Phys. B752, 80 (2006), hep-ph/0512360.

[49] P. S. Bhupal Dev, R. Franceschini, and R. N. Mohapatra, Phys. Rev. D86, 093010 (2012), 1207.2756.

[50] A. Das and N. Okada, Phys. Rev. D88, 113001 (2013), 1207.3734.

[51] F. F. Deppisch, N. Desai, and J. W. F. Valle, Phys. Rev. D89, 051302 (2014), 1308.6789.

[52] A. Das, P. S. Bhupal Dev, and N. Okada, Phys. Lett. B735, 364 (2014), 1405.0177.

[53] A. Das and N. Okada (2015), 1510.04790.

[54] S. Antusch and O. Fischer, JHEP 05, 053 (2015), 1502.05915.

[55] J. A. Casas and A. Ibarra, Nucl. Phys. B618, 171 (2001), hep-ph/0103065.

[56] S. P. Martin, Phys. Rev. D65, 116003 (2002), hep$\mathrm{ph} / 0111209$.

[57] G. Aad et al. (ATLAS), Phys. Rev. D90, 052004 (2014), 1406.3827.

[58] C. Collaboration (CMS) (2014).

[59] P. Bechtle, S. Heinemeyer, O. Stl, T. Stefaniak, and G. Weiglein, JHEP 11, 039 (2014), 1403.1582.

[60] G. W. Anderson and L. J. Hall, Phys. Rev. D45, 2685 (1992).

[61] J. R. Espinosa and M. Quiros, Phys. Lett. B305, 98 (1993), hep-ph/9301285.
[62] J. Choi and R. R. Volkas, Phys. Lett. B317, 385 (1993), hep-ph/9308234.

[63] J. McDonald, Phys. Lett. B323, 339 (1994).

[64] S. W. Ham, Y. S. Jeong, and S. K. Oh, J. Phys. G31, 857 (2005), hep-ph/0411352.

[65] A. Ahriche, Phys. Rev. D75, 083522 (2007), hep$\mathrm{ph} / 0701192$.

[66] J. R. Espinosa and M. Quiros, Phys. Rev. D76, 076004 (2007), hep-ph/0701145.

[67] S. Profumo, M. J. Ramsey-Musolf, and G. Shaughnessy, JHEP 08, 010 (2007), 0705.2425.

[68] L. Dolan and R. Jackiw, Phys. Rev. D9, 3320 (1974).

[69] S. Weinberg, Phys. Rev. D9, 3357 (1974).

[70] M. E. Carrington, Phys. Rev. D45, 2933 (1992).

[71] V. A. Kuzmin, V. A. Rubakov, and M. E. Shaposhnikov, Phys. Lett. B155, 36 (1985).

[72] M. E. Shaposhnikov, Nucl. Phys. B287, 757 (1987).

[73] A. I. Bochkarev and M. E. Shaposhnikov, Mod. Phys. Lett. A2, 417 (1987).

[74] A. Ahriche and S. Nasri, Phys. Rev. D83, 045032 (2011), 1008.3106.

[75] A. Ahriche and S. Nasri, Phys. Rev. D85, 093007 (2012), 1201.4614.

[76] A. Ahriche and S. Nasri, JCAP 1307, 035 (2013), 1304.2055.

[77] A. Ahriche, G. Faisel, S.-Y. Ho, S. Nasri, and J. Tandean, Phys. Rev. D92, 035020 (2015), 1501.06605.

[78] A. Ahriche, K. L. McDonald, and S. Nasri, Phys. Rev. D92, 095020 (2015), 1508.05881.

[79] e. a. H. Baer, available at: http://lcsim.org/papers/DBDPhysics.pdf (????).

[80] J. M. Cline and K. Kainulainen, JCAP 1301, 012 (2013), 1210.4196.

[81] G. Blanger, F. Boudjema, A. Pukhov, and A. Semenov, Comput. Phys. Commun. 192, 322 (2015), 1407.6129.

[82] A. Alloul, N. D. Christensen, C. Degrande, C. Duhr, and B. Fuks, Comput. Phys. Commun. 185, 2250 (2014), 1310.1921. 